\title{
Observation of transverse quadrupolar tune shifts in the Photon Factory storage ring
}

\author{
Shogo Sakanaka, Toshiyuki Mitsuhashi, and Takashi Obina \\ Institute of Materials Structure Science, High Energy Accelerator Research Organization (KEK), 1-1 Oho, \\ Tsukuba, Ibaraki 305-0801, Japan
}

(Received 13 January 2005; published 14 April 2005)

\begin{abstract}
The frequencies (tunes) of transverse quadrupolar oscillations were measured in the $2.5-\mathrm{GeV}$ Photon Factory storage ring at the High Energy Accelerator Research Organization (KEK). As a result, remarkable shifts in the quadrupolar frequencies with the bunch current were found; they were both positive and negative in the horizontal and vertical directions, respectively. This result can be explained by a focusing effect due to short-range wakefields containing a quadrupolar component.
\end{abstract}

DOI: $10.1103 /$ PhysRevSTAB.8.042801

PACS numbers: 29.27.Bd, 29.20.Dh

\section{INTRODUCTION}

In many storage rings, betatron tunes for coherent dipolar (barycentric) oscillations depend on the beam intensity. These tune shifts give us information on the transverse wake force, or on the transverse impedance, of the storage rings [1].

When a bunched beam passes through an axially symmetrical structure with a transverse offset, an induced dipolar wakefield kicks the bunch in the direction of the offset. This generally results in negative tune shifts in both the horizontal and vertical planes. If the structure is not axially symmetrical, on the other hand, the wake force is a superposition of uniform (monopolar), dipolar, quadrupolar, and higher components [2], even when the beam center passes the center of the structure. The wake force can then focus or defocus succeeding particles by its quadrupolar component [2-4]. Nonnegligible effect due to the quadrupolar wakes was recognized in the CERN SPS [5], where the tune shift in the horizontal plane was partly canceled, while that in the vertical plane was enhanced. We expect that the focusing effect due to the quadrupolar wakes can be observed directly by measuring the coherent frequencies of the quadrupolar (bunch shape) oscillations, because these oscillations do not couple with the dipolar wakes. Such a measurement will help us to understand the coupling impedance of the storage rings.

The transverse quadrupolar tunes have been measured [6] in some proton or heavy-ion machines because it is very useful for investigating the space-charge force. However, such a measurement has never been carried out in the electron storage rings. We previously developed a technique [7] for inducing quadrupolar oscillations in an electron storage ring, and then measured the quadrupolar tunes as a function of the beam current per bunch. In this paper we first describe how the quadrupolar wakes affect the dipolar and the quadrupolar tunes. Next, we present the results of the measurements, which were briefly reported in $[8,9]$.

\section{TUNE SHIFTS DUE TO SHORT-RANGE WAKEFIELDS}

When an imaginary bunch, having a finite longitudinal size and infinitesimal transverse sizes, passes through some structure of the storage ring with a transverse offset of $\mathbf{r}_{b}$, a test particle having a transverse offset of $\mathbf{r}$ can be kicked by the wakefield. The transverse wake potential due to this structure is defined by

$$
\begin{aligned}
\mathbf{W}_{\perp}\left(\mathbf{r}_{b}, \mathbf{r}, z\right)= & \frac{1}{q_{\mathrm{b}}} \int_{-\infty}^{\infty} d s\left[\mathbf{E}\left(\mathbf{r}_{b}, \mathbf{r}, s, t=\frac{s-z}{c}\right)\right. \\
& \left.+\mathbf{v} \times \mathbf{B}\left(\mathbf{r}_{b}, \mathbf{r}, s, t=\frac{s-z}{c}\right)\right]_{\perp}
\end{aligned}
$$

where $z$ is the longitudinal position of the test particle relative to the bunch ( $z>0$ denotes a forward position), $s$ is the longitudinal coordinate, $q_{b}$ is the bunch charge, $c$ is the speed of light, $\mathbf{v}$ is the velocity of the particle (we assume $|\mathbf{v}|=c$ ), and $\mathbf{E}$ and $\mathbf{B}$ are the induced electric and magnetic fields, respectively. Assuming that the structure is symmetrical about a horizontal $(x, s)$ plane, we can approximate that the horizontal wake potential $W_{x}$ depends only on the horizontal offsets of $\left(x_{b}, x\right)$ and $z$ [2].

We consider the horizontal motion of a single particle in a bunch having finite transverse sizes. The equation of motion under the wake force is given by

$$
\begin{aligned}
\frac{d^{2} x(z, s)}{d s^{2}}+K_{x}(s) x(z, s)= & \frac{q_{b} e}{E} \delta_{p}\left(s-s_{1}\right) \\
& \times \int_{-\infty}^{\infty} W_{x}\left(x_{b}, x, z\right) f\left(x_{b}\right) d x_{b},
\end{aligned}
$$

where $x(z, s)$ is the horizontal position of the particle, $K_{x}(s)$ is the focusing force due to a ring lattice, $E$ is the particle energy, $e$ is the charge of the particle, $\delta_{p}(s)$ is the periodic delta function having a period of the ring circumference, and $f\left(x_{b}\right)$ is the normalized particle distribution along the horizontal direction. We have assumed the same distribution function, $f\left(x_{b}\right)$, for any $z$, by which a head-tail motion 
was excluded. Only a single wake source at position $s_{1}$ has been included.

In Eq. (2), the horizontal motion is coupled to the longitudinal one through the wake force. We simplify this problem by taking an averaged wake force over the longitudinal particle distribution, which yields

$$
\begin{aligned}
\frac{d^{2} x(s)}{d s^{2}}+K_{x}(s) x(s)= & \frac{q_{b} e}{E} \delta_{p}\left(s-s_{1}\right) \int_{-\infty}^{\infty} \lambda(z) d z \\
& \times \int_{-\infty}^{\infty} W_{x}\left(x_{b}, x, z\right) f\left(x_{b}\right) d x_{b},
\end{aligned}
$$

where $\lambda(z)$ is the normalized longitudinal distribution of particles. Approximating $W_{x}\left(x_{b}, x, z\right)$ by its Taylor series,

$$
\begin{aligned}
W_{x}\left(x_{b}, x, z\right) \approx & W_{x}(0,0, z)+\left(\frac{\partial W_{x}}{\partial x_{b}}\right)_{(0,0, z)} x_{b} \\
& +\left(\frac{\partial W_{x}}{\partial x}\right)_{(0,0, z)} x,
\end{aligned}
$$

we can approximate Eq. (3) by

$$
\begin{aligned}
\frac{d^{2} x(s)}{d s^{2}}+K_{x}(s) x(s)= & \frac{q_{b} e}{E} \delta_{p}\left(s-s_{1}\right) \\
& \times\left[k_{x}^{(0)}+k_{x}^{(1)}\langle x\rangle+k_{x}^{(2)} x\right],
\end{aligned}
$$

with

$$
\begin{aligned}
k_{x}^{(0)} & =\int_{-\infty}^{\infty} W_{x}(0,0, z) \lambda(z) d z, \\
k_{x}^{(1)} & =\int_{-\infty}^{\infty}\left(\frac{\partial W_{x}}{\partial x_{b}}\right)_{(0,0, z)} \lambda(z) d z, \\
k_{x}^{(2)} & =\int_{-\infty}^{\infty}\left(\frac{\partial W_{x}}{\partial x}\right)_{(0,0, z)} \lambda(z) d z,
\end{aligned}
$$

where $\langle x\rangle$ denotes the horizontal position of the bunch centroid. The above $k_{x}^{(0)}, k_{x}^{(1)}$, and $k_{x}^{(2)}$ can be interpreted as the kick factors for the uniform, dipolar and quadrupolar components, respectively.

When there are many structures in the ring, the tune shift for the coherent dipolar oscillation is given by

$$
\delta \nu_{x}^{d}=-\frac{q_{b} e}{4 \pi E} \sum_{j} \beta_{x}\left(s_{j}\right)\left[k_{x, j}^{(1)}+k_{x, j}^{(2)}\right],
$$

where $j$ denotes the index of the structure, $\beta_{x}\left(s_{j}\right)$ is the horizontal betatron function at the location of the $j$ th structure, and $k_{x, j}^{(1)}$ and $k_{x, j}^{(2)}$ are the kick factors for the $j$ th structure. The dipolar tune shift is proportional to the sum of the kick factors for the dipolar and quadrupolar components. On the other hand, the tune shift for each individual particle under no coherent oscillations is called the incoherent tune shift, which is given by

$$
\delta \nu_{x}^{\mathrm{inc}}=-\frac{q_{b} e}{4 \pi E} \sum_{j} \beta_{x}\left(s_{j}\right) k_{x, j}^{(2)} .
$$

The tune shift for the coherent quadrupolar oscillation is approximately equal to twice the incoherent shift,

$$
\delta \nu_{x}^{q} \approx 2 \delta \nu_{x}^{\text {inc }},
$$

as long as its amplitude is small. For large-amplitude oscillations, an additional small tune shift will arise from a quadrupolar wakefield induced by the quadrupolar moment of the beam. For the other vertical motion, both dipolar and quadrupolar tune shifts can be represented similarly. The above Eqs. (7)-(9) suggest that both the measurements of dipolar and quadrupolar tune shifts allow us to estimate the kick factors for the dipolar and quadrupolar wakes separately.

\section{MEASUREMENTS}

\section{A. Horizontal quadrupolar tune shift}

The measurement was carried out in the Photon Factory

\begin{tabular}{|c|c|c|}
\hline Parameter & Symbol & Value \\
\hline Beam energy & $E$ & $2.5 \mathrm{GeV}$ \\
\hline Revolution frequency & $f_{r}$ & $1.602904 \mathrm{MHz}$ \\
\hline Harmonic number & $h$ & 312 \\
\hline Horizontal tune $\mathrm{a}^{\mathrm{a}}$ & $\nu_{x}$ & 9.5974 \\
\hline Vertical tune $^{\mathrm{a}}$ & $\nu_{y}$ & 4.2845 \\
\hline Synchrotron tune & $\nu_{s}$ & 0.0149 \\
\hline Natural rms bunch length ${ }^{\mathrm{a}}$ & $\sigma_{z}$ & $10 \mathrm{~mm}$ \\
\hline Horizontal and vertical radiation damping times & $\tau_{x}, \tau_{y}$ & $7.8 \mathrm{~ms}$ \\
\hline
\end{tabular}
(PF) storage ring [10], which is a $2.5-\mathrm{GeV}$ synchrotron light source at KEK. The principal parameters of the storage ring are given in Table I. A single bunch of electrons was stored during the measurement. To avoid complexity due to nonlinear fields, the octupole magnets were turned off. The experimental setup is shown in Fig. 1. To induce horizontal quadrupolar oscillations, we applied quadrupole magnetic fields oscillating at frequencies close to $2 f_{r} \Delta \nu_{x}(\approx 1.915 \mathrm{MHz})$, where $f_{r}$ is the revolution

TABLE I. Parameters of the PF storage ring under the horizontal quadrupolar-tune measurement.

${ }^{\mathrm{a}}$ At low currents. 


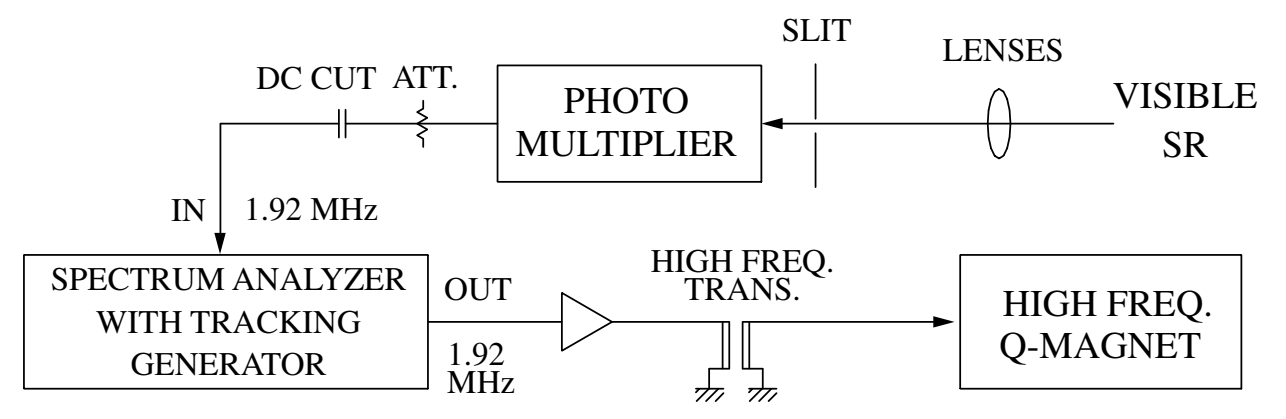

BEAM IMAGE ON THE SLIT

FIG. 1. Setup for measuring the horizontal quadrupolar tunes.

frequency and $\Delta \nu_{x}$ is the fractional horizontal tune. The quadrupole fields were produced using a "high-frequency quadrupole magnet (HFQM)" device [11], which was resonantly driven by a rf amplifier. By applying a horizontal tune modulation of approximately $5.2 \times 10^{-5}$ (at peak), the horizontal quadrupolar oscillation could be induced with an estimated growth time of $3.8 \mathrm{~ms}$.

Excited quadrupolar oscillations were detected using an optical beam-monitor system. Visible synchrotron light from beam line BL-27 was transported to an optical bench, and focused on a slit, producing an image of the beam. Using a photomultiplier (Hamamatsu H5783) behind the slit, the quadrupolar oscillations could be detected as intensity modulations of the synchrotron light. At the same time, we confirmed bunch shape oscillations using a streak camera (Hamamatsu C5680). The gain of the photomultiplier was adjusted so that the amplitudes of the signal were kept almost the same under various beam currents.

The responses of the quadrupolar oscillations were measured by sweeping the excitation frequency of the HFQM

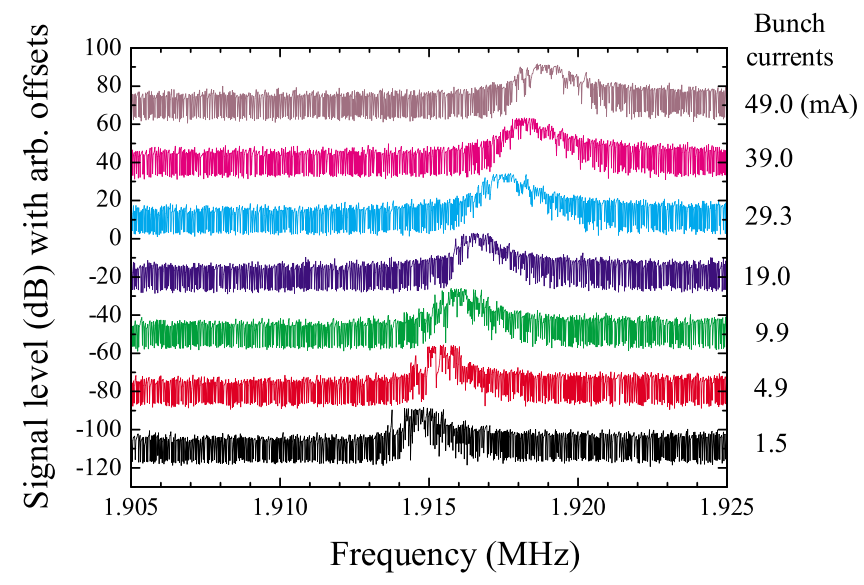

FIG. 2. (Color) Responses of the horizontal quadrupolar oscillation measured under different bunch currents. Abscissa, excitation frequency; ordinate, intensity of the photomultiplier signal. Note that the neighboring traces were separated by additional offsets ( $30 \mathrm{~dB}$ between neighboring traces). The excitation frequency was swept upward. using a spectrum analyzer equipped with a tracking generator. Some of the measured responses under different bunch currents are shown in Fig. 2. Each trace shows a peak where the quadrupolar oscillation was effectively excited. The frequency of each peak can be interpreted as a coherent quadrupolar frequency. At a low bunch current of $1.5 \mathrm{~mA}$, the quadrupolar frequency (approximately $1.9148 \mathrm{MHz}$ ) was very close to twice the fractional horizontal betatron frequency $(957.5 \mathrm{kHz})$ at a low current. At higher currents, the quadrupolar frequency shifted upward, and the peak became wider.

A summary of the measurement is shown in Fig. 3. The horizontal quadrupolar tunes increased almost linearly with the bunch current. A linear fit for these data, except for the first two points, gave a tune slope of $0.048 \mathrm{~A}^{-1}$.

\section{B. Vertical quadrupolar tune shift}

To induce vertical quadrupolar oscillations, we excited the HFQM at frequencies close to $\left(2 \Delta \nu_{y}+3\right) f_{r}$ $(\approx 5.73 \mathrm{MHz})$, where $\Delta \nu_{y}$ is the fractional vertical tune. This frequency was chosen because it fit one of the existing

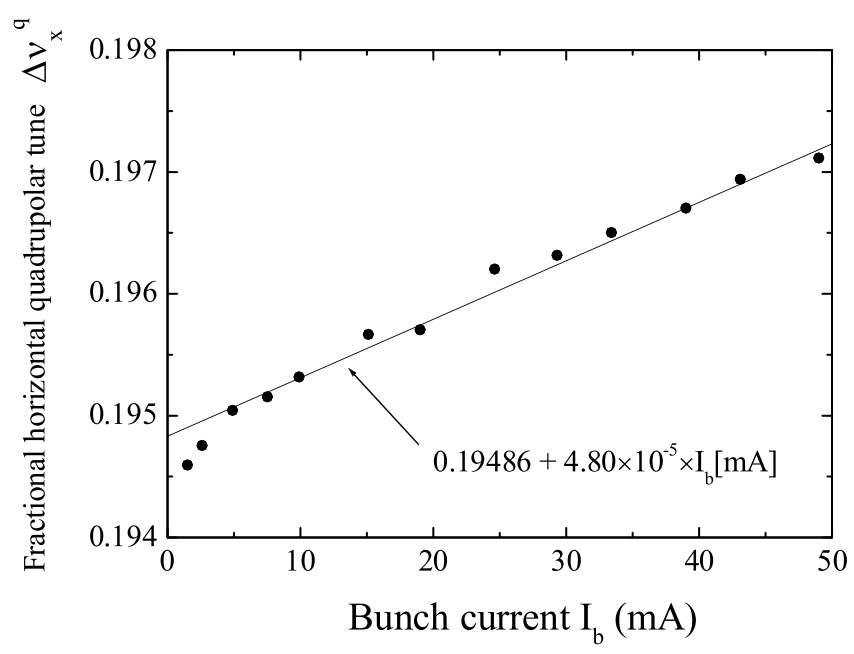

FIG. 3. Measured horizontal quadrupolar tunes as a function of the beam current per bunch. The solid line denotes the linear fit for the data, except for the first two points. 


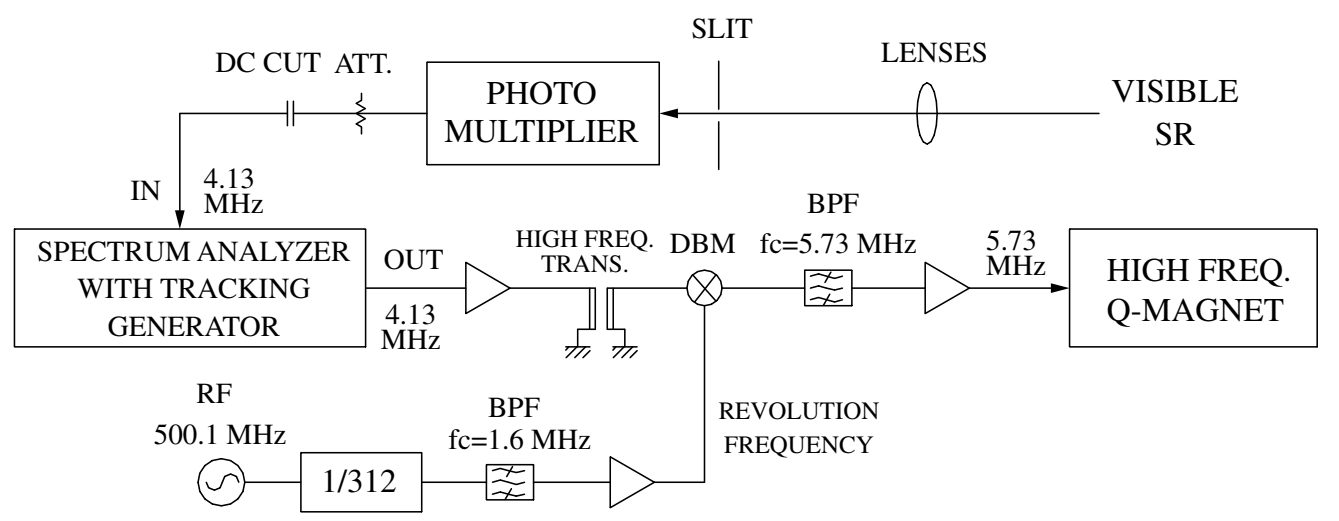

BEAM IMAGE ON THE SLIT

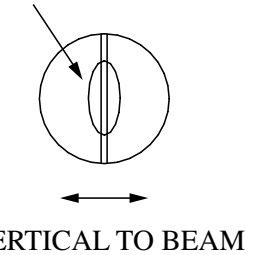

FIG. 4. Setup for measuring the vertical quadrupolar tunes.

power sources. The initial measurement using a similar setup to Fig. 1 indicated that the measurement was considerably affected by rf noise from the HFQM. To avoid this noise, we modified the experimental setup, as shown in Fig. 4.

A sine wave from the tracking generator at a frequency of about $\left(2 \Delta \nu_{y}+2\right) f_{r}(\approx 4.13 \mathrm{MHz})$ was converted up by the revolution frequency, and was used to drive the HFQM. A vertical tune modulation of approximately $7.4 \times 10^{-5}$ was applied, which induced the quadrupolar oscillations with a growth time of about $2.7 \mathrm{~ms}$. Visible synchrotron light was focused onto the slit, where the beam image was rotated by $90^{\circ}$ from that in Fig. 1 . The vertical beam-size oscillations were detected at frequencies of about $\left(2 \Delta \nu_{y}+\right.$ 2) $f_{r}$, which were lower than the excitation frequency by the revolution frequency. The measurement was carried out with a single-bunch beam; the machine parameters were slightly different, as shown in Table II.

We first noticed that the amplitudes of quadrupolar oscillations fluctuated even when the driving frequency was fixed; this was possibly due to a higher sensitivity for the vertical oscillations. When we swept the excitation frequency, we obtained slightly different responses from sweep to sweep due to the fluctuation. An example of the measurement is shown in Fig. 5. Although traces in the lower graph of Fig. 5 varied from sweep to sweep, we could identify in the upper graph a frequency range (roughly 4.131-4.134 MHz) where the quadrupolar oscillations were excited well. We then identified the center of

TABLE II. Storage-ring parameters under the vertical quadrupolar-tune measurement. The other parameters are the same as those in Table I.

\begin{tabular}{lcc}
\hline \multicolumn{1}{c}{ Parameter } & Symbol & Value \\
\hline Revolution frequency $^{c}$ & $f_{r}$ & $1.602883 \mathrm{MHz}$ \\
Horizontal tune $^{\mathrm{a}}$ & $\nu_{x}$ & 9.5988 \\
Vertical tune $^{\mathrm{a}}$ & $\nu_{y}$ & 4.2892 \\
\hline \hline
\end{tabular}

${ }^{\mathrm{a}}$ At low currents. the above range as the most probable quadrupolar frequency. Moreover, we regarded the width of the range as an uncertainty in the measurement.

Figure 6 shows the measured responses of the vertical quadrupolar oscillations at several bunch currents. Ten traces at every current were superposed, where each data point was indicated by a dot. It can be seen that the vertical quadrupolar frequency decreased with the bunch current. Besides, the peaks became faint at high currents.

Figure 7 shows the fractional vertical quadrupolar tunes deduced from the above measurement. The vertical quadrupolar tunes decreased almost linearly with the bunch current. Fitting the data below $35 \mathrm{~mA}$, a tune slope of $-0.076 \mathrm{~A}^{-1}$ was obtained. At low currents, the fractional

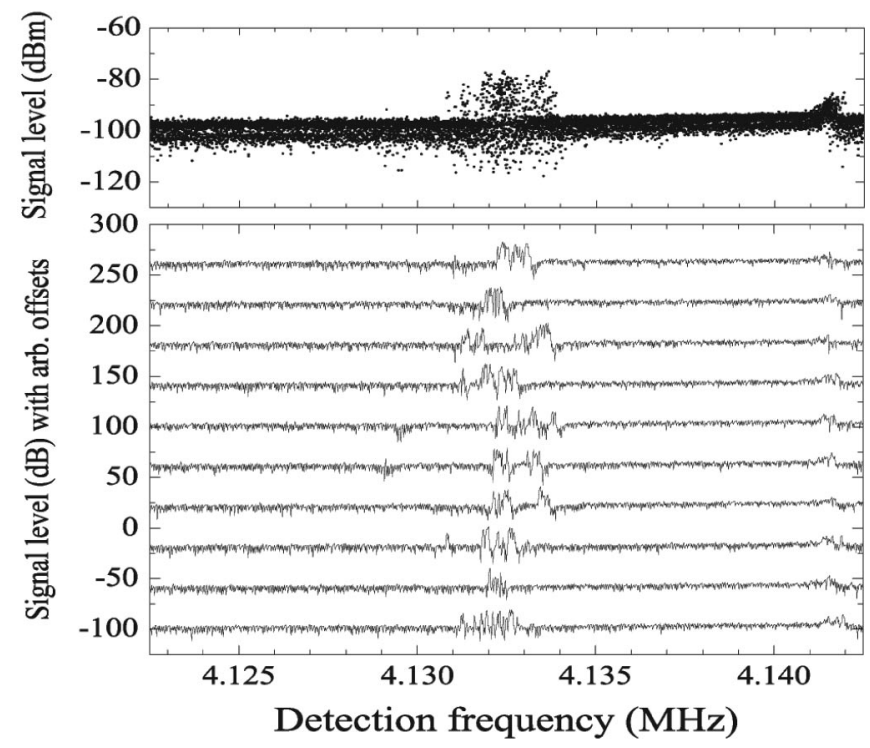

FIG. 5. Responses of the vertical quadrupolar oscillations measured under a bunch current of $10 \mathrm{~mA}$. Lower: ten measured traces which are shown separately by adding offsets (40 dB between neighboring traces). Upper: superposition of the ten traces with the lines between data points omitted. 


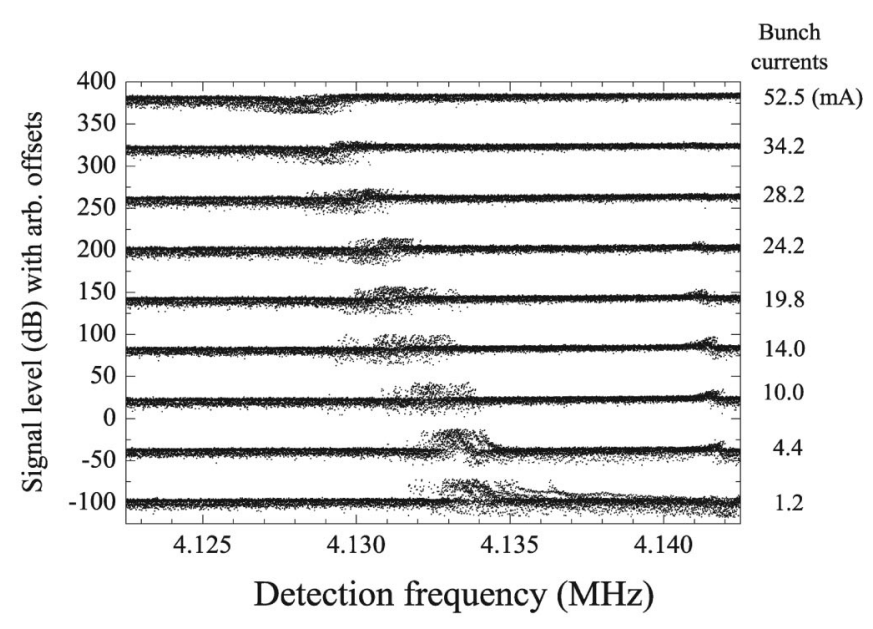

FIG. 6. Responses of the vertical quadrupolar oscillations under several bunch currents. Ten measured traces at every current were superposed. The traces for different currents were separated from each other by adding artificial offsets (60 dB between neighboring traces).

quadrupolar tune (0.5789) was very close to twice the fractional vertical tune (0.2892).

\section{Dipolar tune shifts}

To provide complementary data, we measured the tune shifts for the dipolar oscillations using a conventional rf knockout method. Both Figs. 8 and 9 show the dipolar tune shifts under single-bunch operation, which were measured during the experiment described in the Sec. III B. Fitting these data below a bunch current of $40 \mathrm{~mA}$, we obtained dipolar tune shifts of $-0.013 \mathrm{~A}^{-1}$ (in horizontal) and $-0.11 \mathrm{~A}^{-1}$ (in vertical), respectively.

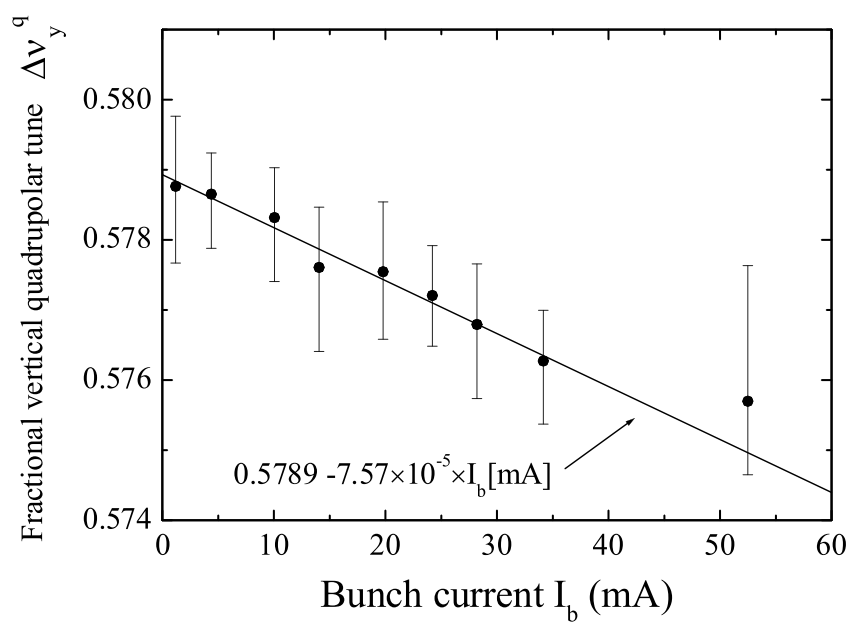

FIG. 7. Measured fractional tunes for the vertical quadrupolar oscillations as a function of the bunch current. Each closed circle indicates the most probable quadrupolar tune. The error bars indicate the uncertainty in the measurement.

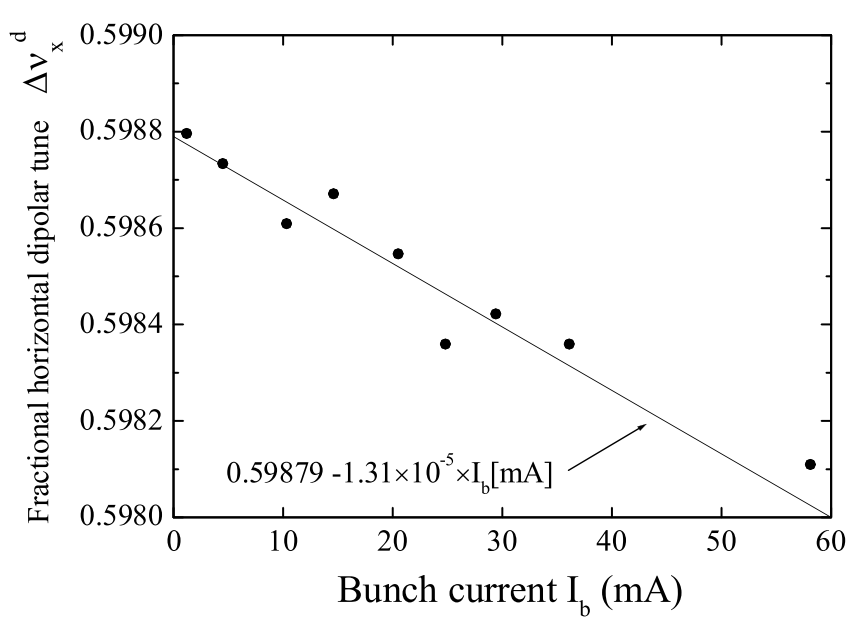

FIG. 8. Measured fractional horizontal tunes of the dipolar oscillations as a function of the beam current per bunch under single-bunch operation.

We also measured the dipolar tune shifts under multibunch operation. While uniformly filling 280 bunches among 312 buckets, we measured the dipolar tunes at beam currents ranging from 10 to $400 \mathrm{~mA}$. A preliminary measurement indicated that the horizontal dipolar tune depended on the total current by $0.002 \mathrm{~A}^{-1}$, while the vertical dipolar tune depended on it by $-0.003 \mathrm{~A}^{-1}$. We found that the dependences of dipolar tunes on the total beam current were much smaller than those on the bunch current.

\section{DISCUSSIONS}

Measurements at the PF storage ring showed that (1) the quadrupolar tunes shifted with the bunch current, (2) the signs of the tune slopes were opposite for the horizontal

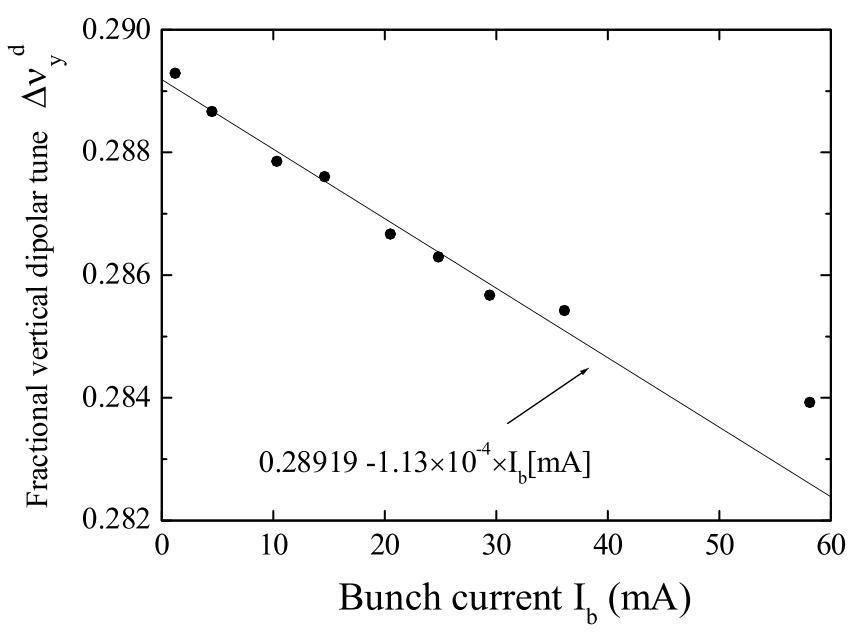

FIG. 9. Measured fractional vertical tunes of the dipolar oscillations as a function of the beam current per bunch under singlebunch operation. 
TABLE III. Summary of the measured tune shifts under single-bunch operation.

\begin{tabular}{lrc}
\hline \hline & Horizontal & \multicolumn{1}{c}{ Vertical } \\
\hline Dipolar tune shift & $-0.013 \mathrm{~A}^{-1}$ & $-0.11 \mathrm{~A}^{-1}$ \\
Quadrupolar tune shift & $0.048 \mathrm{~A}^{-1}$ & $-0.076 \mathrm{~A}^{-1}$ \\
\hline \hline
\end{tabular}

and vertical directions, and (3) the dipolar tunes depended only slightly on the total current under multibunch operation. A summary of the measured tune shifts is given in Table III. These results suggest that some focusing effect should have been induced by the bunch itself. As a candidate, we can list such effects as (i) the quadrupolar component of the short-range wakefield, (ii) the quadrupolar component of the long-range resistive wakefield in noncircular vacuum chambers [12], and (iii) trapped ions.

The above second effect is prominent under multibunch high-current operations, such as in the case of PEP-II [12]. In our experiment, this effect should have contributed little to the quadrupolar tune shifts, because the dipolar tunes depended only slightly on the total current. We also exclude the third possibility because ions should produce focusing forces in both planes. Therefore, the short-range wakefield is the most promising cause for the quadrupolar tune shifts.

According to the depiction given in Sec. II, the dipolar and the quadrupolar tune shifts due to the short-range wakes are given by Eqs. (7) and (9), respectively. Using these relations, we estimated the dipolar and quadrupolar kick factors from the measured tune shifts. To simplify our analysis, we did not take account of the bunch lengthening with the bunch current; the kick factors were then assumed to be constants. Table IV shows the estimated products of the kick factors and the betatron functions. Using average betatron functions of $\left\langle\beta_{x}\right\rangle \sim 3.1 \mathrm{~m}$ and $\left\langle\beta_{y}\right\rangle \sim 6.9 \mathrm{~m}$, total kick factors were roughly estimated to be $k_{x}^{(1)} \sim$ $610 \mathrm{~V} \mathrm{pC}^{-1} \mathrm{~m}^{-1}$ and $k_{x}^{(2)} \sim-390 \mathrm{~V} \mathrm{pC}^{-1} \mathrm{~m}^{-1}$ in the horizontal direction, and $k_{y}^{(1)} \sim 520 \mathrm{~V} \mathrm{pC}^{-1} \mathrm{~m}^{-1}$ and $k_{y}^{(2)} \sim$ $280 \mathrm{~V} \mathrm{pC}^{-1} \mathrm{~m}^{-1}$ in the vertical direction, respectively.

The beam ducts of the PF storage ring are mainly made up of three regular types: a bending-magnet duct (inner aperture $140 \mathrm{~mm}$ wide by $53 \mathrm{~mm}$ high) and two quadrupole-magnet ducts (inner apertures $146 \mathrm{~mm}$ wide by $70 \mathrm{~mm}$ high and $100 \mathrm{~mm}$ wide by $53 \mathrm{~mm}$ high). Other beam ducts for six narrow-gap insertion devices, for two rf sections, and for diagnostic or other devices, are connected to the regular ducts through tapered transitions. The primary source of the impedance would be these transitions, as well as four rf cavities and about 50 unshielded bellows. Because of too many components, we have not carried out a complete estimation of the kick factors by calculations. However, the above kick factors are reasonable regarding the following points. First, the signs of $k_{x}^{(2)}$ and $k_{y}^{(2)}$, which indicate focusing in the horizontal direction and
TABLE IV. Products of the kick factors and the betatron functions estimated from the measured tune shifts.

\begin{tabular}{lr}
\hline \hline$\sum_{j} \beta_{x}\left(s_{j}\right) k_{x, j}^{(1)}$ & $1.9 \times 10^{15} \mathrm{~V} / \mathrm{C}$ \\
$\sum_{j} \beta_{x}\left(s_{j}\right) k_{x, j}^{(2)}$ & $-1.2 \times 10^{15} \mathrm{~V} / \mathrm{C}$ \\
$\sum_{j} \beta_{y}\left(s_{j}\right) k_{y, j}^{(1)}$ & $3.6 \times 10^{15} \mathrm{~V} / \mathrm{C}$ \\
$\sum_{j} \beta_{y}\left(s_{j}\right) k_{y, j}^{(2)}$ & $1.9 \times 10^{15} \mathrm{~V} / \mathrm{C}$ \\
\hline \hline
\end{tabular}

defocusing in the vertical direction, are consistent with our calculation for a typical flat structure. Second, the ratio $k_{x}^{(2)} / k_{y}^{(2)} \sim-1.4$ is close to the theoretical restriction of -1 .

In Fig. 2, the peak of the quadrupolar frequency becomes wider at high currents. The reason can be either a spread in the incoherent frequency or an increase in the damping rate, due to some collective effect. This issue is also worth investigating further.

\section{CONCLUSIONS}

The tunes of transverse quadrupolar oscillations were measured in the PF electron storage ring as a function of the bunch current. As a result, both positive and negative tune shifts were found for the horizontal and vertical oscillations, respectively. This result can be basically explained by a self-focusing effect of the bunch due to a quadrupolar component of short-range wakefields, which was induced in axially asymmetrical structures. We showed that by measuring both dipolar and quadrupolar tune shifts one can estimate the kick factors for the dipolar and quadrupolar wakes separately.

\section{ACKNOWLEDGMENTS}

We wish to thank members of the PF storage ring for their support.

[1] See, for example, D. Rice, K. Adams, M. Billing, E. Blum, R. Littauer, B. McDaniel, R. Meller, D. Morse, S. Peck, S. Peggs, J. Seeman, R. Siemann, R. Talman, M. Tigner, and E. vonBorstel, IEEE Trans. Nucl. Sci. 28, 2446 (1981); J. C. Denard, J. Le Duff, M. P. Level, P. C. Marin, E. M. Sommer, and H. Zyngier, ibid. 28, 2474 (1981); R. Klatt, R. D. Kohaupt, and T. Weiland, ibid. 32, 2356 (1985).

[2] S. Heifets, A. Wagner, and B. Zotter, SLAC Report No. AP110, 1998.

[3] A. Wagner and B. Zotter, in Proceedings of the Fifth European Particle Accelerator Conference, Sitges, Spain, 1996 (Institute of Physics, Bristol, UK, 1996), pp. 1256-1258.

[4] Bruno W. Zotter and Semyon A. Kheifets, Impedances and Wakes in High-Energy Particle Accelerators (World Scientific, Singapore, 1998).

[5] J. Gareyte, in Proceedings of the Eighth European Particle Accelerator Conference, Paris, 2002 (EPS-IGA and 
CERN, Geneva, 2002), pp. 89-93; H. Burkhardt, G. Rumolo, and F. Zimmermann, in Proceedings of the Eighth European Particle Accelerator Conference, Paris, 2002, pp. 1449-1451.

[6] M. Chanel, in Proceedings of the Fifth European Particle Accelerator Conference, Sitges, Spain, 1996 (Ref. [3]), pp. 1015-1017; R. Bär, I. Hofmann, P. Moritz, and U. Oeftiger, Nucl. Instrum. Methods Phys. Res., Sect. A 415, 460 (1998); T. Uesugi, S. Machida, Y. Mori, M. Ikegami, S. Ninomiya, A. Mochihashi, T. Oki, S. Hidaka, D. Arakawa, T. Toyama, and K. Noda, in Proceedings of the 1999 Particle Accelerator Conference, New York, 1999 (IEEE, Piscataway, NJ, 1999), pp. 1821-1823.

[7] S. Sakanaka, Y. Kobayashi, T. Mitsuhashi, and T. Obina, in Proceedings of the 2001 Particle Accelerator Conference, Chicago, 2001 (IEEE, Piscataway, NJ, 2001), pp. 393-395; S. Sakanaka, Y. Kobayashi, T. Mitsuhashi, and T. Obina, Jpn. J. Appl. Phys. 42, 1757 (2003).
[8] S. Sakanaka, T. Mitsuhashi, and T. Obina, in Proceedings of the 2003 Particle Accelerator Conference, Portland, OR, 2003 (IEEE, Piscataway, NJ, 2003), pp. 3207-3209.

[9] S. Sakanaka, T. Mitsuhashi, and T. Obina, in Proceedings of the Ninth European Particle Accelerator Conference, Luzerne, 2004 (EPS-AG and CERN, Geneva, 2004), pp. 1702-1704.

[10] S. Asaoka et al., in Proceedings of the Second Asian Particle Accelerator Conference, Beijing, 2001 (Institute of High Energy Physics, Beijing, 2001), pp. 69-73; Photon Factory Activity Report 2003, KEK Progress Report 2004-1, edited by J. Adachi, T. Honda, T. Iwazumi, R. Kato, M. Kawasaki, H. Kawata, T. Kosuge, K. Mase, H. Maezawa, Y. Misumi, F. Mori, H. Sugiyama, M. Tanaka, N. Tomita, and N. Usami, 2004.

[11] S. Sakanaka, T. Mitsuhashi, A. Ueda, and M. Izawa, Nucl. Instrum. Methods Phys. Res., Sect. A 325, 1 (1993).

[12] A. Chao, S. Heifets, and B. Zotter, Phys. Rev. ST Accel. Beams 5, 111001 (2002). 